\title{
Endoscopic Ultrasound-Guided Antitumor Therapy for Advanced Pancreatic Cancer
}

Pancreatic cancer is a tumor with an unfavorable prognosis with surgical resection being the only effective therapy that prolongs the survival. The response to systemic chemotherapy is suboptimal, and this limited response to systemic chemotherapy is partly due to poor tissue penetration of drugs consequent to intense tumor desmoplasia. ${ }^{[1]}$ Moreover, localized chemotherapy is always preferred over systemic chemotherapy as it limits the systemic toxicity of drugs along with increasing its local concentration. Endoscopic ultrasound-guided fine-needle injection (EUS-FNI) of chemotherapeutic drugs is an appealing method of delivering localized chemotherapy. Anatomic location of the pancreas just behind the stomach makes it easily accessible by EUS, and suboptimal response to systemic chemotherapy makes pancreatic cancer an ideal disease to be treated with EUS-guided injection therapy. It is hypothesized that as the chemotherapeutic agent is injected in the interstitial space of the tumor, its concentrations are much greater than that developed after systemic injection, thereby probably overriding the chemotherapeutic protective mechanisms developed by the cancer cells. ${ }^{[1]}$ Despite this exciting postulated mechanism, the experience with EUS-FNI of chemotherapeutic drugs in pancreatic cancer is not so encouraging. ${ }^{[2-5]}$ Therefore, various alternative approaches such as development and testing of drugs that are related to cancer tissue microenvironments such as hyaluronidase, gamma-secretase inhibitor, sonic hedgehog inhibitor, antibodies against CD40, and connective tissue growth factor are being considered as treatment approaches. ${ }^{[6]}$

Carbohydrate sulfotransferase 15 (CHST15) and its specific product chondroitin sulfate-E have been shown to be associated with poor prognosis in many cancers including pancreatic cancers. ${ }^{[7,8]}$ Suppression of CHST15 by siRNA has been shown to inhibit tumor growth in mice. ${ }^{[7,8]}$ Thus, CHST15 is a novel therapeutic target that regulates the tumor microenvironment in pancreatic cancer. STNM01 is a synthetic double-stranded RNA oligonucleotide that selectively inhibits the expression of CHST1511 and is a novel therapeutic agent for pancreatic cancer. In this news and views, we discuss an interesting study that evaluated the safety and efficacy of EUS-guided FNI of STNM01 in patients with unresectable pancreatic cancer. ${ }^{[8]}$

Six patients with histological confirmed pancreatic adenocarcinoma by EUS-guided fine-needle aspiration (EUS-FNA), the Eastern Cooperative
Oncology Group performance status of 0 or 1 , age $>20$ years, and the diagnosis as "progressive disease" after previous chemotherapy based on the Response Evaluation Criteria in Solid Tumors (RECIST) guidelines were studied in this single-center, nonrandomized, open study of STNM01 (Stelic Institute and Co., Inc., Tokyo, Japan). A 16-ml STNM01 was administered into the tumor under EUS guidance using a conventional 22-gauge needle (Expect, Boston Scientific, Japan) at 16 different sites in the tumor (1 $\mathrm{ml}$ at each site).

The patients were discharged from the hospital $24 \mathrm{~h}$ after EUS-FNI and were followed up 2 and 4 weeks later. If patients tolerated the initial injection, further doses of STNM01 were given after 4 weeks of observation. Treatment was repeated every month until disease progression, as defined by the RECIST guidelines, was not observed. EUS-FNA was also performed along with repeated EUS-FNI with the tumor samples stained with hematoxylin and eosin as well as immunostained for CHST15. Five immunostained images from each sample were captured at $\times 200$, and the intensity of immunostaining was analyzed using ImageJ $1.50 \mathrm{i}$ with an average intensity of each image being used for the final analyses.

The median number of injections given was 3 (range: 2-8) with the drug STNM01 being well tolerated in all patients, and no adverse effects were noted. The mean plasma concentration of STNM01 at $2 \mathrm{~h}$ after injection was below the detection limit of $0.5 \mathrm{ng} / \mathrm{mL}$ in all the studied patients. Following initial EUS-FNI, the median tumor size decreased from 31 to $29 \mathrm{~mm}$ with tumor necrosis being observed in four patients $(66.6 \%)$. The mean serum-soluble CD44 variant $6(\mathrm{sCD} 44 \mathrm{v} 6)$ levels also significantly decreased from 98.1 to $83.2 \mathrm{ng} / \mathrm{mL}$, with improvement in pain in majority of patients. However, median serum CA19-9 level did not change significantly. The median survival was 5.8 months (range: 2.0-18.0 months). There was $50 \%$ decrease of CHST15 expression following EUS-guided FNI. The authors concluded that EUS-FNI of oligonucleotide STNM01 in pancreatic cancer is safe and feasible and CHST15 reduction could predict tumor progression and survival.

\section{COMMENTARY}

Despite advances in medicine, the prognosis of pancreatic cancer remains dismal, and all the newer 
chemotherapeutic agents have failed to either significantly improve survival or the resectability rates. EUS-FNI is a new and promising treatment modality for pancreatic cancer. Hypothetically, increasing the intratumoral chemotherapy levels could lead to increase in the local effects of chemotherapy, augment the radiation effect as well as limit the adverse effects. Previously, EUS-FNI has been used for intratumoral injection of T-lymphocytes (cytoimplant) in eight patients with a median survival of 13.2 months. ${ }^{[9]}$ However, subsequent Phase II/III trial of cytoimplant was prematurely terminated as response rates and survival were greater for patients randomized to gemcitabine as well as some studies reporting severe drug-related sepsis. ${ }^{[1,9]}$ EUS-guided FNI of ONYX-015, a gene-deleted replication-selective adenovirus that preferentially kills malignant cells, has been reported with modest response. ${ }^{[10]}$ A pilot study of EUS-FNI of dendritic cells reported no major adverse effects with a median survival of 9.9 months. ${ }^{[11]}$

EUS-FNI of TNFerade, a replication-deficient adenoviral vector containing the human TNF-a gene that expresses tumor necrosis factor- $\alpha$ under the control of the Egr-1 promoter, which is inducible by chemotherapy and radiation, has also been reported with encouraging results. $^{[2]}$ More recently, EUS-FNI with gemcitabine reported encouraging results with a median survival of 10.4 months (2.7-68) and four (20\%) patients with Stage III unresectable disease being downstaged and subsequently successfully undergoing $\mathrm{R} 0$ resection. ${ }^{[1]}$

The current study demonstrated that STNM01 suppressed the CHST15 expression in cancer cells and thus possibly reversing invasion and improving survival. CD44 is a marker for pancreatic cancer stem cells (CSCs) and correlates with poor survival, and following EUS-FNI, the mean serum-sCD44v6 levels significantly decreased from 98.1 to $83.2 \mathrm{ng} / \mathrm{mL}$, thereby suggesting therapeutic response. Despite the limitations of small sample size as well as single-center study, this study is a significant advancement in the treatment of pancreatic cancer, and further studies with higher dose of STNM01 as well as larger sample size randomized studies are needed to confirm these initial encouraging results. Although encouraging, the present study also has the same limitations as of previous studies on EUS-FNI, namely, small size and lack of control arm. Therefore, further studies with large sample size as well as control arm are needed to establish the best EUS-FNI agent, its dose, and long-term therapeutic effect and also study the long-term adverse effects.

Surinder Singh Rana

Department of Gastroenterology, Postgraduate Institute of Medical Education and Research, Chandigarh, India

Address for correspondence: Dr. Surinder Singh Rana, Department of Gastroenterology, Postgraduate Institute of Medical Education and Research, Sector 12, Chandigarh - 160 012, India. E-mail: drsurinderrana@yahoo.co.in

\section{REFERENCES}

1. Levy MJ, Alberts SR, Bamlet WR, Burch PA, Farnell MB, Gleeson FC, et al. EUS-guided fine-needle injection of gemcitabine for locally advanced and metastatic pancreatic cancer. Gastrointest Endosc 2017;86:161-9.

2. Hecht JR, Farrell JJ, Senzer N, Nemunaitis J, Rosemurgy A, Chung $\mathrm{T}$, et al. EUS or percutaneously guided intratumoral TNFerade biologic with 5-fluorouracil and radiotherapy for first-line treatment of locally advanced pancreatic cancer: A phase I/II study. Gastrointest Endosc 2012;75:332-8.

3. Han J, Chang KJ. Endoscopic ultrasound-guided direct intervention for solid pancreatic tumors. Clin Endosc 2017;50:126-37.

4. Luz LP, Al-Haddad MA, Sey MS, DeWitt JM. Applications of endoscopic ultrasound in pancreatic cancer. World J Gastroenterol 2014;20:7808-18.

5. Sharma V, Rana SS, Bhasin DK. Endoscopic ultrasound guided interventional procedures. World J Gastrointest Endosc 2015;7:628-42.

6. Neesse A, Krug S, Gress TM, Tuveson DA, Michl P. Emerging concepts in pancreatic cancer medicine: Targeting the tumor stroma. Onco Targets Ther 2013;7:33-43.

7. Ito Z, Takakura K, Suka M, Kanai T, Saito R, Fujioka S, et al. Prognostic impact of carbohydrate sulfotransferase 15 in patients with pancreatic ductal adenocarcinoma. Oncol Lett 2017; 13:4799-805.

8. Nishimura M, Matsukawa M, Fujii Y, Matsuda Y, Arai T, Ochiai Y, et al. Effects of EUS-guided intratumoral injection of oligonucleotide STNM01 on tumor growth, histology, and overall survival in patients with unresectable pancreatic cancer. Gastrointest Endosc 2018;87:1126-31.

9. Chang KJ, Nguyen PT, Thompson JA, Kurosaki TT, Casey LR, Leung EC, et al. Phase I clinical trial of allogeneic mixed lymphocyte culture (cytoimplant) delivered by endoscopic ultrasound-guided fine-needle injection in patients with advanced pancreatic carcinoma. Cancer 2000;88:1325-35.

10. Hecht JR, Bedford R, Abbruzzese JL, Lahoti S, Reid TR, Soetikno RM, et al. A phase I/II trial of intratumoral endoscopic ultrasound injection of ONYX-015 with intravenous gemcitabine in unresectable pancreatic carcinoma. Clin Cancer Res 2003;9:555-61. 
11. Irisawa $\mathrm{A}$, Takagi $\mathrm{T}$, Kanazawa $\mathrm{M}$, Ogata $\mathrm{T}$, Sato $\mathrm{Y}$, Takenoshita S, et al. Endoscopic ultrasound-guided fine-needle injection of immature dendritic cells into advanced pancreatic cancer refractory to gemcitabine: A pilot study. Pancreas 2007;35:189-90.
This is an open access journal, and articles are distributed under the terms of the Creative Commons Attribution-NonCommercial-ShareAlike 4.0 License, which allows others to remix, tweak, and build upon the work non-commercially, as long as appropriate credit is given and the new creations are licensed under the identical terms.

Access this article online

\begin{tabular}{|l|l|}
\hline \multicolumn{2}{|c|}{ Access this article online } \\
\hline Quick Response Code: & \\
& \\
\end{tabular}

How to cite this article: Rana SS. Endoscopic ultrasound-guided antitumor therapy for advanced pancreatic cancer. J Dig Endosc 2018;9:95-7. 